\title{
Distance Evaluation in Pattern Matching Based on Frontier Topological Graph
}

\author{
Gilles d'Andréa \\ LIRMM, 161 rue Ada, 34392 Montpellier Cedex 5 - FranCE \\ $\mathrm{Tel}+33(0) 4.67 .41 .86 .05-\mathrm{Fax}+33(0) 4.67 .41 .85 .00$ \\ email: dandrea@lirmm.fr
}

\begin{abstract}
We focus to an application of control of printing quality in industrial environment. We try to value the quality in the sense of the readability of a text printed in disturbed conditions. We propose in this paper, topological comparison criteria between two superposed patterns, based on a graph representation.

The graph is built according to the regions and their adjacencies in the resulting image of a maximal superposition of two patterns: each edge of the graph is not only joining two regions, but also note a frontier between two regions. The study of this graph highlight creation or loss of connectivity, appearance or disappearance of holes or meaningful part between the compared patterns. This topological study allows us to propose an interesting distance evaluation function between the two patterns to be compared.

keywords: Distance Evaluation Function, Graph Representation, Pattern Matching.
\end{abstract}

\section{Introduction}

In image analysis, pattern matching is often used to recognize a particular element in an image: one of the main application is character recognition. The first step of pattern matching is the extraction from the image of the candidate pattern, which is generally rotated and at a different scale of the model pattern. Then, the extracted pattern and the model pattern are superposed in order to maximize the overlapping surface. Finally, their differences are evaluated to decide their matching.

The problem we would like to solve is to control the printing quality in an industrial environment. This application is different from the classical applications of character recognition in the sense that the characters to be printed are known. In fact, we want to evaluate the readability of a character. So, we propose a distance evaluation function for two patterns based on topological differences criteria. 
From the resulting image of a maximal superposition of two binary patterns, we build a Frontier Topological Graph [Fio96] that gives a topologically consistent representation of the image. This representation allows us to detect topological differences between the two patterns, such as creation or loss of connectivity, appearance or disappearance of hole or meaningful part in patterns. This topological characterization is the basis of the distance evaluation function proposed in this paper.

In the next section, we present the framework of this paper, then we will recall the definition of the Frontier Topological Graph, and how to build it from a superposition of two patterns. At last we show how to compute from the graph, different criteria in order to calculate the proposed distance function.

\section{Problem Definition}

\subsection{Application and Objectives}

We focus on an application of control of printing quality in industrial environment. We try to value the quality in the sense of the readability of a text printed in disturbed conditions with the a priori knowledge of printed characters and patterns.

The image acquisition, the pattern extraction from the image and the research of a better superposition between the printed pattern and the pattern to be printed are out of the scope of this paper (see [GW87,dF97]). We are interested in the comparison methods between a printed pattern and a model pattern to be printed, to value the printing quality. Therefore, we propose in this paper, topological comparison criteria between two patterns relying on a graph representation based on the Frontier Topological Graph (a sort of RAG $[\operatorname{Ros} 74, \mathrm{Pav77]})$ in order to compute a distance between the two patterns to be compared.

\subsection{Topological Comparison of two Binary Patterns}

Pattern matching in an image is a difficult problem. Indeed, images are generally noisy, and researched pattern in the image could have undergone transformations like scaling, rotation or slant. So, these distortions forbid a matching only based on a pixel to pixel comparison between the extracted pattern and its model.

Methods of pattern matching get out relatively well with scaling and rotation problems and determine generally a better superposition of the two patterns in the sense of surface [GW87,Vos92]. Then, their geometric differences are generally valued by a function of distance minimized to validate the matching. 
In this paper, we are interested in the geometric comparison step of two binary patterns. We present a distance function between two patterns, robust to the possible imperfections of the previous step of superposition.

The main idea is to combine the images of the two patterns in a superposition maximizing the overlapped surface, and from this image, we construct a Frontier Topological Graph according to the different regions of the resulting image: common regions to both patterns, regions of first or second pattern and empty regions corresponding to the image background. In this graph, each edge is not only joining two regions, but also denotes a frontier between two regions.

So, the study of this graph allows us to highlight creation or loss of connectivity, appearance or disappearance of holes or meaningful parts of the pattern, and remains robust to the image noise and induced slight geometric distortions of the extraction step. We are now going to recall the definition of an FTG, and to study how to construct it and what its properties are, allowing us to characterize the topological differences between the two patterns.

\section{Frontier Topological Graph}

The Frontier Topological Graph (FTG for short) is a tcpologically consistent representation for images in image analysis. It is based on the star-topology described in [AAF95] and presents several advantages sn Region Adjacency Graph (RAG: [Ros74,Pav77]), such as number and relative positions of frontiers between regions or inclusion of regions. The FTG is a planar multi-graph derivated from a combinatorial map model. Thus, each edge is not only joining two regions, but also denotes a frontier between two regions (see Figure 1).

\subsection{Definition}

So an FTG, $G(V, D, \alpha, \sigma)$, where $V$ is the set of vertices of the graph, $D$ is the set of darts (half-edges), $\sigma$ an involution on $D$ and $\alpha$ a permutation on $D$, has the following properties:

1. Each region is represented by one and only one vertex.

¿. A dart (half-edge) $e$ is always incident to a vertex $R$ of $V$. The notation $e^{R}$ denotes the incident vertex of $e$.

3. An edge of the graph is a non-ordered pair $(e, \sigma(e))$ where $e$ belongs to $D$. Let $E$ be the set of edges, $\left(e_{1}^{R}, e_{2}^{k}\right) \in E$ if and only if there exists a frontier between $R$ and $R^{\prime}$. Thus an edge represents a frontier. A dart symbolizes the frontier "as seen from" the region to which it is incident. 


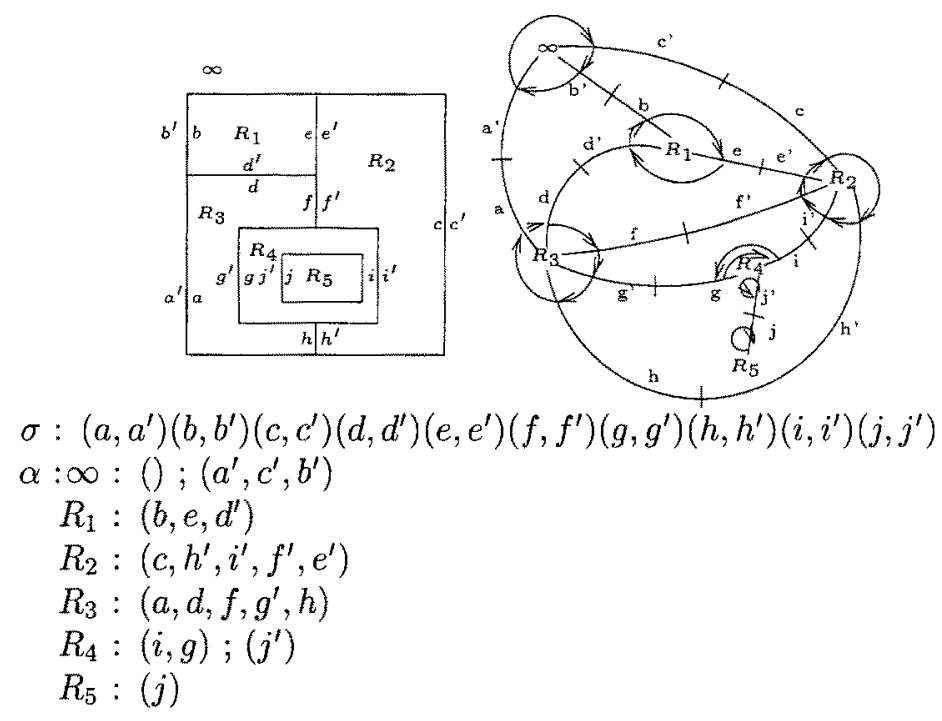

Fig. 1. Example of an FTG and its algebraic representation

4. The cycles of the permutation $\alpha$ on $D$ correspond to a contour of a given region and respect the order induced by the sequence of frontiers making the contour.

5. $\infty$ is a particular vertex of the graph symbolizing the exterior of the image.

6. To each vertex is associated the list of the cycles of $\alpha$ related to the contours of the region represented by this vertex. By convention, the exterior contour will always be the first of the list.

For a more complete definition of the FTG, we refer to Christophe Fiorio's PhD. thesis [Fio95], and [Fio96].

\subsection{FTG Building}

Given $P_{A}$ and $P_{B}$, two patterns for wich we want to evaluate a given superposition. Given $I$ the resulting image of this superposition such as pixels coming solely from the pattern $P_{A}$ (resp. solely from the pattern $P_{B}$, from both patterns $P_{A}$ and $P_{B}$, from the background) are noted $A$ (resp. $B, C, E$ ).

In this image $I$, we define the regions or 4 -connected ${ }^{1}$ components of pixels, according to their label $A, B, C$ or $E$. Then, we build $G(V, D, \alpha, \sigma)$ the FTG associated to $I$ with the algorithm of extraction presented in [Fio96] (see Figure 2).

${ }_{1}$ Two pixels are 4-connected if their coordinates differ by one in only one direction. 

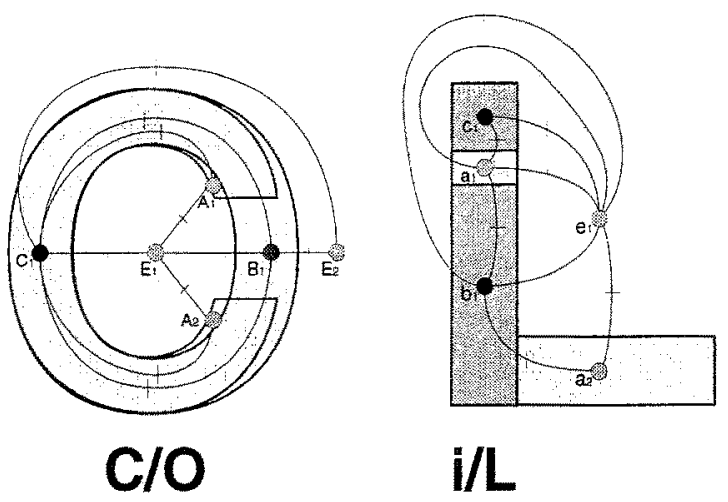

Fig. 2. Examples of Superposed Characters and their associated FTG.

We complete $G$ in labeling each vertex of $v$, with the surface in pixels of the associated region, and each edge of $D$, with the length in pixcls of the associated frontier.

\subsection{FTG Preprocessing}

Noise in the images of the patterns $P_{A}$ and $P_{B}$ may generate mistakes in the construction of the FTG. Indeed, each connected component of interference pixels forms a useless and cumbersome vertex in the FTG. But these vertices may be easily detected on criteria of region size and positions and then be eliminated.

Given $s_{n}$, an arbitrary threshold bounding the surface of connected components generated by noise in the image. For every vertex $x$ of $G$ with a smaller surface than $s_{n}, x$ will be withdrawn of $G$, if $x$ satisfies one of the following conditions:

- $x$ is a pendant vertex of the graph, linked to a single vertex $y$ of $G$ : region of $x$ is entirely contained by region of $y$. In this case, region of $x$ is integrated to region of $y$, and $x$ disappears from the graph.

- $x$ is linked to two vertices $y$ and $z$ such that only $z$ is a region labeled $E$ : region $x$ is assimilated to noise at frontier of region $y$.

The noise elimination in the FTG need not in any case, modify the topology of the image: the deletion of a region considered as noise need not create a connection between two regions labeled $A, B$ or $C$ that were not previously connected. 


\section{Topological Characterization Based on the FTG}

Given $I$ the resulting image of the superposition of two patterns $P_{A}$ and $P_{B}$, we define $G(V, D, \alpha, \sigma)$ an FTG where $V$ is the set of vertices of the graph, $D$ is the set of darts (half-edges), $\sigma$ an involution on $D$ and $\alpha$ a permutation on $D$. Each vertex of $V$ is also respectively labeled $A, B, C$ or $E$ according to the label of the corresponding region.

The superposition of $P_{A}$ on $P_{B}$ and the study of the associated FTG allows us to evaluate quantitatively and qualitatively the topological differences between $P_{A}$ and $P_{B}$.

\subsection{Study of the FTG}

Regions $A$ and $B$ denote differences between the two patterns. In order to characterize them, we are going to focus on vertices labeled $A$ or $B$. The table below summarizes according to the adjacency of vertices labeled $A$ or $B$, the topological differences such as creation or loss of connectivity, appearance or disappearance of a hole, as well as extension or reduction of a meaningful part of the pattern.

\begin{tabular}{c||c|c|c|c||l} 
Vertex & links to $A$ & links to $B$ & links to $C$ & links to $E$ & Characterization \\
\hline \hline$x \in A$ & 0 & - & $\geq 2$ & - & Creation of connectivity \\
\hline$x \in A$ & 0 & - & 0 & 1 & Appearance of a connected comp. \\
\hline$x \in A$ & 0 & - & 1 & 0 & Disappearance of a hole \\
\hline$x \in A$ & 0 & - & 1 & $\geq 1$ & Extension of pattern \\
\hline \hline$x \in B$ & - & 0 & $\geq 2$ & - & Loss of connectivity \\
\hline$x \in B$ & - & 0 & 0 & 1 & Disappearance of a connected comp. \\
\hline$x \in B$ & - & 0 & 1 & 0 & Appearance of a hole \\
\hline$x \in B$ & - & 0 & 1 & $\geq 1$ & Reduction of pattern
\end{tabular}

Creation/Loss of connectivity: The topology of a character may be deeply modified by creation or loss of connectivity between two regions. In the example of Figure 2, the vertex $A_{1}$ creates a connectivity that closes the character " $\mathrm{C}$ " to transform it into " $\mathrm{O}$ ", and the vertex $a_{1}$ merges the vertical bar of the " $\mathrm{i}$ " with its point.

Appearance/Disappearance of a connected component: Characters " $\mathrm{i}$ " and " $\mathrm{j}$ " are formed of two connected components, the disappearing of their points would be detected by a vertex labeled $A$ solely linked to a vertex labeled $E$. 
Appearance/Disappearance of a hole: In a disturbed printing, appearance or disappearance of a hole may be generated by a lack or an excess of ink. In fact, characters are generally too thin to undergo this kind of transformations. On the other hand a general pattern may be modified, so a circle may become a disk and vice versa.

Extension/Reduction of pattern: Detection of extension or reduction of the surface of a pattern needs particular attention. Indeed, this modification of the surface may be meaningful or not. In the example of Figure 2, the vertices $A_{1}$ and $A_{2}$ may be disregarded, on the other hand the vertex $a_{2}$ modifies the geometry of the character in creating the horizontal bar of the capital L.

The study of the ratio between the surface of the concerned region and the length of its frontier allows us to define the geometric importance of this region. In this way, we must define a critical threshold for the ratio surface/frontier of a region.

In the case of $\mathrm{i} / \mathrm{L}$ example, the region $a_{2}$ has a great ratio surface/frontier, therefore the character underwent an extension of surface modifying meaningfully its geometry. In the case of the $\mathrm{C} / \mathrm{O}$ example, vertices $A_{1}$ and $A_{2}$ have a small ratio surface/frontier that allows us to consider these regions as the result of a difference of thickness of character or a slight scale, rotation or slant.

\subsection{Distance Evaluation and Applications}

The topological characterizations that brings us the study of the FTG for a maximal superposition of two patterns, allows in weighting each of these characterizations to compute a distance function between the patterns. So, we can use this function in order to value the printing quality of a text. Nevertheless this function may also be used to compare two different patterns, so it may be used in an application of character recognition where its needed to minimize the distance function to match an extracted pattern in a model patterns set.

Besides, this function of distance evaluation may be computed efficiently: the extraction of the Frontier Topological Graph is achieved in linear time of the image size [Fio96], and the analysis of the FTG is achieved in studying the direct adjacency of each vertex of the graph. So, these topological comparison criteria are therefore efficient to compute.

\section{Conclusion}

We defined an interesting method for evaluating a topological distance between two binary patterns. For a given superposition and the resulting image of this 
superposition, we build a Frontier Topological Graph relative to four different types of regions of the image: regions where the two patterns do superpose, regions belonging only to the first or the second pattern, and regions corresponding to the image background.

The study of the FTG allows us to evaluate the topological differences between the two patterns, by detecting creation or loss of connectivity, appearing or disappearing of holes or meaningful parts in the patterns. A preprocessing on the FTG and a fine analysis of surfaces and frontiers of regions allows us to minimize problems due to image noise, and slight differences of scaling, of rotation and of alignment between the two patterns.

This work is directly concerned with a control of quality of disturbed printing. The proposed method is used to quantify the readability of printed text, but it may also apply to a distance function in character recognition or in binary pattern matching.

\section{References}

[AAF95] Ehoud Ahronovitz, Jean-Pierre Aubert, and Christophe Fiorio. The startopology: a topology for image analysis. In $5^{\text {th }}$ Discrete Geometry for Computer Imagery, Proceedings, pages 107-116. Groupe GDR PRC/AMI du CNRS, september 1995.

[dF97] Gilles d'Andréa and Christophe Fiorio. Maximal superposition of grids and an application. In $7^{\text {th }}$ International Workshop, DGCI'97, number 1347 in Lecture Notes in Computer Sciences, pages 63-71, Montpellier, France, December 1997.

[Fio95] Christophe Fiorio. Approche interpixel en analyse d'images : une topologie et des algorithmes de segmentation. Thèse de doctorat, Université Montpellier II, 24 novembre 1995.

[Fio96] Christophe Fiorio. A topologically consistent representation for image analysis: the frontiers topological graph. In $6^{\text {th }}$ International Workshop, DGCI'96, number 1176 in Lecture Notes in Computer Sciences, pages 151-162, Lyon, France, November 1996.

[GW87] Rafael C. Gonzalez and Paul Wintz. Digital image processing. AddisonWesley, second edition, 1987.

[Pav77] Theo Pavlidis. Structural Pattern Recognition. Springer-Verlag, New York, 1977.

[Ros74] Azriel Rosenfeld. Adjacency in digital pictures. Inform. and Control, 26:24$33,1974$.

[Vos92] George Vosselman. Relational Matching. Lecture Notes in Computer Science. Springer-Verlag, Berlin Heidelberg Germany, 1992. 